\title{
Associations between alcohol use and accelerated biological ageing
}

\author{
Sunniva M. K. Bøstrand ${ }^{1,2}$ (]) | Kadi Vaher ${ }^{1,3}$ @ | Laura de Nooij $^{1}$ | \\ Matthew A. Harris ${ }^{1}$ | James H. Cole ${ }^{4,5,6}$ | Simon R. Cox ${ }^{7}$ | Riccardo E. Marioni ${ }^{8}$ | \\ Daniel L. McCartney ${ }^{8}$ | Rosie M. Walker ${ }^{8,9}$ | Andrew M. Mclntosh ${ }^{1}$ | \\ Kathryn L. Evans $^{8}$ | Heather C. Whalley ${ }^{1}$ | Robyn E. Wootton ${ }^{10,11}$ | Toni-Kim Clarke ${ }^{1}$ \\ ${ }^{1}$ Division of Psychiatry, Royal Edinburgh Hospital, The University of Edinburgh, Edinburgh, UK \\ ${ }^{2}$ Centre for Regenerative Medicine, Institute for Regeneration and Repair, The University of Edinburgh, Edinburgh, UK \\ ${ }^{3}$ MRC Centre for Reproductive Health, The Queen's Medical Research Institute, The University of Edinburgh, Edinburgh, UK \\ ${ }^{4}$ Centre for Medical Image Computing, Department of Computer Science, University College London, London, UK \\ ${ }^{5}$ Institute of Psychiatry, Psychology and Neuroscience, King's College London, London, UK \\ ${ }^{6}$ Dementia Research Centre, Institute of Neurology, University College London, London, UK \\ ${ }^{7}$ Department of Psychology, The University of Edinburgh, Edinburgh, UK \\ ${ }^{8}$ Centre for Genomic and Experimental Medicine, Institute of Genetics and Molecular Medicine, The University of Edinburgh, Edinburgh, UK \\ ${ }^{9}$ Centre for Clinical Brain Sciences, The University of Edinburgh, Edinburgh, UK \\ ${ }^{10} \mathrm{MRC}$ Integrative Epidemiology Unit, University of Bristol, Bristol, UK \\ ${ }^{11}$ School of Psychological Science, University of Bristol, Bristol, UK
}

\section{Correspondence}

Heather C. Whalley, Division of Psychiatry, Royal Edinburgh Hospital, The University of Edinburgh, Morningside Park, Edinburgh EH10 $5 \mathrm{HF}$, UK.

Email: heather.whalley@ed.ac.uk

Sunniva M. K. Bøstrand, Centre for Regenerative Medicine, Institute for Regeneration and Repair, The University of Edinburgh, 5 Little France Drive, Edinburgh EH16 4UU, UK.

Email: sunniva.bostrand@ed.ac.uk

\section{Funding information}

Alzheimer's Research UK, Grant/Award Number: ARUK-PG2017B-10; Brain and Behavior Research Foundation, Grant/Award Number: 27404; Chief Scientist Office of the Scottish Government Health Directorates, Grant/Award Number: CZD/16/6; Medical Research Council, Grant/Award Numbers: MC_UU_00011/1, MC_UU_00011/3, MR/ R024065/1; National Institutes of Health,

\begin{abstract}
Harmful alcohol use is a leading cause of premature death and is associated with agerelated disease. Biological ageing is highly variable between individuals and may deviate from chronological ageing, suggesting that biomarkers of biological ageing (derived from DNA methylation or brain structural measures) may be clinically relevant. Here, we investigated the relationships between alcohol phenotypes and both brain and DNA methylation age estimates. First, using data from UK Biobank and Generation Scotland, we tested the association between alcohol consumption (units/week) or hazardous use (Alcohol Use Disorders Identification Test [AUDIT] scores) and accelerated brain and epigenetic ageing in 20,258 and 8051 individuals, respectively. Second, we used Mendelian randomisation (MR) to test for a causal effect of alcohol consumption levels and alcohol use disorder (AUD) on biological ageing. Alcohol use showed a consistent positive association with higher predicted brain age (AUDIT-C: $\beta=0.053, p=3.16 \times 10^{-13}$; AUDIT-P: $\beta=0.052$, $p=1.6 \times 10^{-13}$; total AUDIT score: $\beta=0.062, p=5.52 \times 10^{-16}$; units/week:
\end{abstract}


Grant/Award Number: R01AG054628; Scottish Funding Council, Grant/Award Number: HR03006; UK Research and Innovation, Grant/Award Number: MR/ R024790/2; Wellcome Trust, Grant/Award Numbers: 104036/Z/14/Z, 108890/Z/15/Z $\left.\beta=0.078, p=2.20 \times 10^{-16}\right)$, and two DNA methylation-based estimates of ageing, GrimAge (units/week: $\beta=0.053, p=1.48 \times 10^{-7}$ ) and PhenoAge (units/week: $\left.\beta=0.077, p=2.18 \times 10^{-10}\right)$. MR analyses revealed limited evidence for a causal effect of AUD on accelerated brain ageing ( $\beta=0.118, p=0.044)$. However, this result should be interpreted cautiously as the significant effect was driven by a single genetic variant. We found no evidence for a causal effect of alcohol consumption levels on accelerated biological ageing. Future studies investigating the mechanisms associating alcohol use with accelerated biological ageing are warranted.

\section{KEYWORDS}

alcohol use, brain ageing, epigenetic ageing, Generation Scotland, Mendelian randomisation, UK Biobank

\section{1 | INTRODUCTION}

Harmful alcohol use is a leading cause for premature death globally. ${ }^{1}$ Excessive alcohol use affects multiple tissues ${ }^{2}$ and is associated with an increased risk for all-cause mortality ${ }^{3}$ and age-related diseases including diabetes, liver diseases and dementia. ${ }^{4}$ A recent large-scale epidemiological investigation has challenged the view that moderate alcohol consumption can be beneficial, showing that even small amounts of alcohol negatively impact on health. ${ }^{1}$ Ageing itself is a complex process of progressive deterioration due to the accrual of cellular damage over time, ${ }^{5}$ and rates of these age-associated biological processes vary between individuals. This could account for some of the variation in susceptibility to age-related disease and suggests that measures of biological ageing may be more clinically relevant than chronological age., Several biomarkers have been proposed to measure individual variation in biological ageing, including those based on DNA methylation (DNAm) or brain magnetic resonance imaging (MRI). To date, studies investigating associations between alcohol use and biological ageing have been limited to small sample sizes, report inconsistent findings and have not probed the potential causality of these associations.

Recent analytical approaches, such as that developed by Cole et al., ${ }^{6}$ use machine learning algorithms trained to predict chronological age from brain structural MRI data. Testing these algorithms on new structural data produces a metric of brain age that correlates strongly with chronological age, although its deviation from chronological age reflects accelerated/decelerated brain ageing. ${ }^{8}$ Accelerated brain ageing predicts mortality in older adults and correlates with cognitive and physical decline. ${ }^{9}$ Several epigenetic clocks have also been used to characterise biological ageing and are hypothesised to capture molecular processes involved in declining tissue function. ${ }^{10}$ These biomarkers use weighted averages of methylation levels at specific cytosine-phosphate-guanine (CpG) sites to produce estimates of epigenetic age. Similarly to brain age, a greater positive deviation in DNAm age from chronological age predicts all-cause mortality ${ }^{11}$ and has been linked to a range of age- and lifestyle-related health outcomes, including exercise and diet, ${ }^{12}$ and cognitive ability and decline. ${ }^{13,14}$
Previous work has associated alcohol use with the acceleration of biological ageing, as measured by both brain and DNAm ageing. ${ }^{12,15-19}$ Ning et al. ${ }^{15}$ showed that more frequent consumption of alcohol was associated with a higher brain age relative to peers, with the lowest brain age found in individuals who reported drinking only occasionally. Higher alcohol intake frequency was also associated with an older-appearing brain using multiple MRI modalities to predict brain age. ${ }^{16}$ Similarly, higher levels of alcohol consumption across 30 years of follow-up were associated with reductions in grey matter density and white matter structural integrity, suggesting that heavy alcohol consumption may lead to accelerated brain ageing. ${ }^{2}$

Alcohol use is associated with variation in $\mathrm{DNAm}^{20}$; thus, investigations into the associations between alcohol use and epigenetic ageing could allow insight into the shared molecular mechanisms underlying harmful alcohol use and ageing. Previous studies have reported complex relationships between alcohol consumption levels and epigenetic ageing, with studies showing positive, ${ }^{18}$ negative ${ }^{12}$ and non-linear ${ }^{19}$ associations. Additionally, a recent study suggests both positive and negative genetic associations between various alcoholrelated traits and epigenetic ageing using LD score regression. ${ }^{21}$

Cross-sectional, observational epidemiological studies are prone to bias from residual confounding and reverse causation, limiting the investigations of cause-effect relationships. Genome-wide association studies (GWASs) have identified several loci implicated in both the clinical diagnosis of alcohol use disorder (AUD) and varying alcohol consumption levels. ${ }^{22,23}$ These genetic variants can be utilised as instruments to determine the most likely direction of effect between a modifiable exposure, alcohol use and the outcome, biological ageing, using Mendelian randomisation (MR). ${ }^{24}$ It follows the logic that if a modifiable exposure (e.g. alcohol use) is the cause of an outcome (e.g. accelerated biological ageing), then individuals with genetic variants predisposing them towards increased alcohol use should be more likely to experience accelerated biological ageing.

Here, we investigated the relationship between alcohol use and biological ageing using the largest brain imaging $(N=20,258)$ and DNAm ( $N=8051)$ datasets to date. We hypothesised that higher levels of alcohol consumption would associate with both higher 
DNAm age and brain age and that these associations would reflect a causal effect of higher alcohol use on accelerated biological ageing.

\section{2 | METHODS AND MATERIALS}

The UK Biobank (UKB) and the Generation Scotland: Scottish Family Health Study (GS:SFHS) cohort data were used in the current study. For a graphical representation of the samples and data available in both of the cohorts for the current analyses, see Figure S1.

\section{1 | Study populations: UKB}

UKB comprises $N=502,617$ individuals recruited from across the United Kingdom. ${ }^{25}$ UKB received ethical approval from the National Health Service (NHS) National Research Ethics Service North West (reference: 11/NW/0382). The present study was carried out under UKB project ID 4844. At the time of writing, we used the latest available UKB neuroimaging (see protocol in Miller et al. ${ }^{25}$ ) release consisting of $N=21,386$ individuals. After removing extreme outliers (defined as MRI measurements $>5 * \mathrm{SD}$ from the mean), cases of image acquisition problems and excluding previous or never drinkers, $N=20,258$ individuals were included in the present study (see Table S1 and Figure S2 for demographics and sample selection).

\subsection{1 | Alcohol consumption}

Lifestyle measures were collected at baseline and online follow-up. Alcohol use in units/week at baseline was calculated by converting the sum of reported average weekly intake of red wine, champagne plus white wine, beer plus cider, spirits, fortified wine and other alcoholic drinks into alcohol units. At online follow-up, a subset of participants ( $N=14,710)$ completed the Alcohol Use Disorders Identification Test (AUDIT), a 10-item screening tool developed by the World Health Organisation ${ }^{26}$ to assess alcohol consumption and alcohol-related behaviours and problems. To examine the relationship between alcohol use and brain age, we used four measures of alcohol consumption: alcohol units/week and three measures from the AUDIT questionnaire-a composite score of alcohol consumption (AUDIT-C; sum of Questions 1-3), problematic alcohol use (AUDIT-P; sum of Questions 4-10) and the total score across all items (AUDIT-T).

\subsection{2 | Brain age estimates}

We utilised a measure of brain age derived from structural T1-weighted MRI data as described by Cole et al. ${ }^{6}$ and implemented using the brainageR software package (https://github.com/jamescole/brainageR). In the sample of current drinkers, the correlation between brain age and chronological age was $r=0.734$, $p<2.2 \times 10^{-16}$ (full demographics in Table S1). This measure was subsequently residualised over chronological age, sex, imaging site (Manchester/Newcastle) and scanner head position X, Y and Z coordinates. The residualised brain age measure reflected deviation of brain age from chronological age (controlling for aforementioned covariates), with positive values representing accelerated brain ageing.

\section{2 | Study populations: Generation Scotland}

GS:SFHS cohort comprises $\sim 24,000$ individuals aged $\geq 18$ years at recruitment and is described in detail elsewhere. ${ }^{27}$ At baseline, participants were assessed for a range of health, demographic and lifestyle factors and provided samples for DNA extraction. GS:SFHS has been granted ethical approval from the NHS Tayside Committee on Medical Research Ethics, on behalf of the NHS (reference: 05/S1401/89) and has Research Tissue Bank status (reference: 15/ES/0040). The present study includes individuals for whom information about alcohol consumption, smoking, body mass index (BMI) and DNAm data (profiled in two sets; see below) was acquired at baseline (full demographics in Table S2).

\subsection{1 | Alcohol consumption}

We used self-reported units/week as a quantification of a person's alcohol consumption. The present study includes individuals who reported being current drinkers; extreme outliers (defined as participants with alcohol consumption in units/week $>4^{*}$ standard deviations [SD] from the mean) were additionally excluded from the analysis. For a comparison between the GS:SFHS participants included and not included in the current study, see Table S14.

\subsection{2 | DNAm profiling}

Whole blood genomic DNA samples were treated with sodium bisulphite using the EZ-96 DNA Methylation Kit (Zymo Research, Irvine, California), following the manufacturer's instructions. Genomewide DNAm was profiled using the Infinium MethylationEPIC BeadChip (Illumina Inc., San Diego, California) in accordance with the manufacturer's protocol. DNAm was profiled in 9778 participants across two processing sets (Set $1 \mathrm{~N}=5190$ [comprised related individuals], Set $2 N=4583$ [comprised unrelated (to each other and to Set 1) individuals]). Quality control and normalisation was carried out separately in the two sets using standard methods. Arrays were scanned using a HiScan scanner (Illumina Inc., San Diego, California), with initial inspection of array quality carried out in GenomeStudio v2011.1. Additional quality control measures were implemented as described in detail previously. ${ }^{28}$ Briefly, outlier sites and participants, together with participants for whom there was a mismatch between their predicted sex (based on DNAm data) and their recorded sex, were excluded from both sets; samples were then normalised (separately) using the Dasen method from the watermelon ${ }^{29} \mathrm{R}$ package. The 
final DNAm dataset comprised data for 5087 individuals in Set 1 and 4450 individuals in Set 2. Participant data in the two sets were analysed separately and then meta-analysed. After applying exclusion criteria (see above), the current study included 4260 participants from Set 1 and 3791 participants from Set 2 (full demographics in Table S2).

\subsection{3 | Epigenetic estimates of age}

Epigenetic age and age acceleration measures were calculated using the online age calculator (https://dnamage.genetics.ucla.edu/) developed by Horvath. ${ }^{10}$ Normalised DNAm beta-values were submitted to the calculator using the 'Advanced Analysis for Blood Data' option. Four DNAm-based estimates of age were calculated, namely, Hannum $^{30}$ and Horvath ${ }^{10}$ epigenetic age, DNAm GrimAge ${ }^{31}$ and DNAm PhenoAge ${ }^{32}$ (see below), which all strongly correlated with chronological age (Table S3) and with one another (Table S15). From these estimates, four age-adjusted epigenetic age acceleration (EAA) measures (intrinsic and extrinsic EAA [IEAA and EEAA, respectively], ${ }^{33}$ AgeAccelGrim $^{31}$ and AgeAccelPheno ${ }^{32}$ ) were calculated (see below). The age acceleration measures were uncorrelated with chronological age (Table S3). In addition, the resulting EAA measures were only weakly to moderately correlated with one another (Table S15), suggesting they capture different aspects of DNAm ageing.

The Horvath epigenetic age was calculated based on methylation levels at $353 \mathrm{CpG}$ sites following the approach developed by Horvath. ${ }^{10}$ Hannum epigenetic age was calculated based on DNAm levels at the $71 \mathrm{CpGs}$ identified by Hannum. ${ }^{30}$ From these DNAm age estimates, we derived two measures for epigenetic age acceleration that are either independent of blood cell counts or enhanced by changes in blood cell composition, as described previously. ${ }^{33}$ In brief, intrinsic epigenetic age acceleration (IEAA) is defined as the residual term of a multivariate model regression estimated Horvath methylation age on chronological age, adjusting for blood cell counts estimated from the methylation data (naive CD8 $+\mathrm{T}$ cells, exhausted CD8 $+\mathrm{T}$ cells, plasmablasts, CD4+ T cells, natural killer cells, monocytes and granulocytes), and is therefore by definition independent of blood immune cell counts. Extrinsic epigenetic age acceleration (EEAA), on the other hand, tracks age-related changes in blood cell composition as well as cell-intrinsic epigenetic changes. It is estimated by first calculating Hannum's DNAm age and second increasing the contribution of three cell types whose abundance is known to change with age (naive cytotoxic T cells, exhausted cytotoxic T cells and plasmablasts) by forming a weighted average of Hannum's DNAm age estimate with these three cell type estimates using the Klemera-Doubal approach. ${ }^{34}$ EEAA is defined as the residual term of univariate model regressing the weighted estimated Hannum's epigenetic age in chronological age.

The DNAm GrimAge was developed by Lu et al. ${ }^{31}$ by first creating DNAm-based surrogates for 12 plasma proteins and smoking packyears and thereafter regressing time to death on chronological age, sex and these DNAm-based surrogates. This model selected chronological age, sex and DNAm-based surrogates for smoking pack-years and seven plasma proteins (adrenomedullin, beta-2-microglobulin, cystatin C, growth differentiation factor 15 , leptin, plasminogen activation inhibitor 1 and tissue inhibitor metalloproteinase). The linear combination of these variables is then used to estimate the DNAm GrimAge. Adjusting DNAm GrimAge for chronological age generated the measure of epigenetic GrimAge acceleration, AgeAccelGrim. ${ }^{31}$

The DNAm PhenoAge was developed by Levine et al. $^{32}$ by first regressing ageing-related mortality on 42 clinical markers and chronological age to select variables for inclusion in the phenotypic age (PhenoAge) score. Ten variables (chronological age, albumin, creatinine, glucose and C-reactive protein levels, lymphocyte percentage, mean cell volume, red blood cell distribution width, alkaline phosphatase and white blood cell count) were then used to calculate mortality score, which was subsequently converted into units of years to create the measure of PhenoAge. Thereafter, DNAm from whole blood was used to predict this PhenoAge. This approach generated the selection of $513 \mathrm{CpGs}$, and linear combination of the weighted $\mathrm{CpG}$ s yields the DNAm PhenoAge. ${ }^{32}$ Adjusting DNAm PhenoAge for chronological age generated the measure of phenotypic epigenetic age acceleration, AgeAccelPheno.

\section{3 | Statistical methods}

All statistical analyses were performed in R (Versions 3.3.2, 3.6.1 and 4.0.1). ${ }^{35}$ Scaling by $z$-transformation was applied for all numeric variables in the regression models.

\subsection{1 | Association between alcohol use and brain age in UKB}

The variables AUDIT-C, AUDIT-P, AUDIT total scores and alcohol consumption in units/week were entered separately into linear models to test for association with residualised brain age. Smoking status (coded at baseline as a binary value denoting whether individuals had ever smoked or never smoked), age and sex were added as covariates in each model in order to control for the effects of these variables. As a sensitivity analysis, we additionally adjusted for age of completing full-time education in the regression model (Figure S3). Benjamini-Hochberg correction for false discovery rate (FDR) was applied to the regression models.

\subsection{2 | Association between alcohol consumption and epigenetic age acceleration in GS:SFHS}

Set 1 data (related subset of GS:SFHS) statistical analyses were conducted in ASReml-R Version 3.0 (www.vsni.co.uk/software/asreml) to fit a linear mixed model to control for relatedness within the sample by fitting an inverse relationship matrix derived from pedigree information as a random effect. Set 2 data (unrelated subset of GS:SFHS) was analysed using linear regression $(\mathrm{Im})$ function in base $\mathrm{R}$. In each model, EAA was fit as the dependent variable, and alcohol 
consumption (as $\log _{10}[1+$ units/week $]$ to adjust for non-normal distribution) as the independent variable; sex, BMI, smoking pack-years (at baseline, individuals were asked to self-report their tobacco exposure [cigarettes/day], age when they started smoking and years since stopped smoking, and pack-years variable was calculated as packs [20 cigarettes/pack] smoked per day multiplied by years as a smoker) and inverse relationship matrix fitted as a random effect only for Set 1 in ASReml were added as covariates. A sensitivity analysis was conducted by additionally adjusting the models for years of education (this information is collected from GS:SFHS participants in 10 categories corresponding to $0,1-4,5-9,10-11,12-13,14-15,16-17,18-$ $19,20-21,22-23$, or $24+$ years of education). As smoking is strongly associated with DNAm, further sensitivity analyses were conducted in non-smoking individuals (i.e. who reported never having smoked tobacco): $n=2207$ in Set 1 and $n=1998$ in Set 2. To combine the coefficient estimates from the two sets into a single estimate, we applied an inverse variance-weighted fixed-effects meta-analysis model using the function metagen, implemented in the meta package in $\mathrm{R}^{36}$ FDR correction for multiple testing was applied across all fully adjusted models (fully adjusted regressions in Sets 1 and 2 and metaanalysis) and separately for the two sensitivity analyses. Results were plotted using the function forest in the R package meta.

\section{\begin{tabular}{l|l}
2.4 & Two-sample MR
\end{tabular}}

Two-sample MR analysis was performed in R using the TwoSampleMR package from MRBase ${ }^{37}$ using summary statistics extracted from nonoverlapping GWASs.

\subsection{1 | Exposure GWAS: Alcohol consumption (AUDIT-C) and AUD}

Data on the genetic association with alcohol use were extracted from Kranzler et al. ${ }^{22}$ This study was carried out in the Million Veteran Programme population, which is based in the United States and therefore highly unlikely to overlap with UKB or GS:SFHS populations made up of British and Scottish individuals, respectively. Kranzler et al. ${ }^{22}$ reported a genome-wide significant association between 10 single nucleotide polymorphisms (SNPs) and AUD, as well as 13 SNPs associated with alcohol consumption as measured by AUDIT-C. These SNPs were identified as independent by linkage disequilibrium (LD) clumping using a 500-kb genomic window and $r^{2}<0.1 .^{22}$ Here, we extracted the summary statistics as reported in the European ancestry population (see Tables S1 and S2 in Kranzler et al. ${ }^{22}$ ).

\subsection{2 $\quad$ Outcome GWASs}

Brain age

GWAS for brain age acceleration was performed in a subset of unrelated White British individuals in the UKB imaging cohort. 16,133 individuals were included using a relatedness cut-off of 0.05 . To perform the GWAS, brain age was entered as the outcome variable and age, sex, genotyping array and the 20 first principal components derived from genotype data as covariates. From the results of this analysis, we extracted the summary statistics (beta and standard error of the effect allele of each SNP) for the 10 and 13 SNPs identified as genome-wide significant by Kranzler et al. ${ }^{22}$ for AUD and AUDIT-C, respectively. The full GWAS summary statistics are available here: https://datashare.is.ed.ac.uk/handle/10283/3797. We used the Functional Mapping and Annotation of Genome-Wide Association Studies (FUMA) ${ }^{38}$ SNP2GENE function to extract results from the brain age GWAS. In order to identify independently associated variants, clumpbased pruning was applied in FUMA using an $r^{2}$ of 0.1 and a 1-Mb sliding window using the UKB White British sample as the LD reference panel. See Table S17 for the 20 top hits and Figure S6 for a Manhattan plot of main results. Using the GWAS summary statistics, we calculated the SNP-based heritability (Table S18) and genetic overlap between brain age and 770 other disease traits (Table S19) using LD score regression implemented in the online software LD Hub (http://ldsc.broadinstitute.org/). ${ }^{39}$

\section{Epigenetic age}

Data on the genetic association with AgeAccelGrim and AgeAccelPheno were extracted from McCartney et al. ${ }^{21}$ These GWASs were conducted in 34,962 European ancestry individuals, and a fixed-effects meta-analysis was performed to combine the summary statistics. ${ }^{21}$ Supplementary tables were inspected to ensure that the Million Veteran Programme cohort was not included in these metaGWASs. From the GWAS results (available here: https://datashare.is. ed.ac.uk/handle/10283/3645), we extracted the corresponding summary statistics for the 13 SNPs identified as GW significant for AUDIT-C by Kranzler et al. ${ }^{22}$

For SNPs unavailable in the outcome GWAS summary statistics, proxy SNPs were searched for (https://ldlink.nci.nih.gov/?tab= Idproxy) with a minimum LD $R^{2}=0.9$. In AgeAccelGrim and AgeAccelPheno GWASs, rs185177474 was not available; thus, rs151242810 was used as a proxy $\left(R^{2}=0.976\right)$.

See Tables S4 and S5 for the full MR input testing causal effects of AUDIT-C and AUD, respectively, on biological ageing.

We performed two MR analyses with brain age as the outcome, where AUD or AUDIT-C were the exposures, respectively. One MR analysis was performed with either AgeAccelGrim or AgeAccelPheno as the outcome and AUDIT-C as the exposure. The main MR models included 13 SNPs to probe for the causal effect of AUDIT-C on biological ageing and 10 SNPs to probe for the causal effect of AUD on brain ageing.

We applied complementary two-sample MR methods (inverse variance weighted [IVW], MR-Egger, weighted median and weighted mode-based estimation). IVW was the main analysis with each of the others providing sensitivity analyses, which each make different assumptions about the nature of pleiotropy (where the genetic variant associates with the outcome via an independent pathway to the exposure). Therefore, the strongest evidence for a causal effect would be 
where the estimates from all methods are consistent. To test the suitability of the MR-Egger method, the $I^{2}$ statistic was calculated to quantify the degree of regression dilution bias due to measurement error of SNP-exposure effects. ${ }^{40}$ The mean F-statistic as an indicator of instrument strength was also calculated (Table S7). We additionally calculated the variance explained by the genetic instruments of AUDIT-C and AUD using a modified method as described in ${ }^{41}$ (Table S20). Further, we used the MR-Egger intercept to test for the presence of horizontal pleiotropy (Table S9) and Steiger filtering ${ }^{42}$ to test for the most likely direction of effect (Tables S10-S13) and calculated Cochran's $Q$ to assess heterogeneity suggestive of pleiotropy (Table S8). When there was evidence for a causal effect based on the IVW model, we performed MR-Presso ${ }^{43}$ and Radial $M^{44}$ to detect potential outliers.

\section{$3 \mid$ RESULTS}

\section{1 | Alcohol use associations with brain age in UKB}

Using linear regression, we found a consistent positive relationship between brain age and the four measures of alcohol use (alcohol units/week ( $N=20,258,52.1 \%$ female), AUDIT-C, AUDIT-P and AUDIT-T ( $N=14,710,53.5 \%$ female)) (Figure 1), with higher levels of self-reported alcohol consumption associated with a more advanced brain age. The largest effect was found for alcohol consumption measured in units/week $(\beta=0.078,95 \% \mathrm{Cl}$ [0.066; 0.093], $\left.p<2.20 \times 10^{-16}\right)$. Adjusting for education did not significantly alter the outcome of this analysis for any measure of alcohol use (Figure S3).

\subsection{Alcohol use associations with epigenetic age in GS:SFHS}

Linear regression and fixed-effects meta-analyses were used to investigate associations between alcohol consumption (units/week) and four measures of EAA (IEAA, EEAA, AgeAccelGrim and AgeAccelPheno) in 8051 individuals in total (Set $1 n=4260$ [60.9\% female], Set $2 n=3791$ [55.3\% female]) from the GS:SFHS cohort (full demographics in Table S2). We found a positive association between alcohol consumption and AgeAccelGrim ( $\beta=0.053$ [0.034; 0.071], $\left.p=1.48 \times 10^{-7}\right)$ and AgeAccelPheno $(\beta=0.077$ [0.055; 0.100], $p=2.18 \times 10^{-10}$ ), but not between alcohol consumption and IEAA or EEAA (Figure 2). These results are robust to adjustment for years of education (Figure $\mathrm{S} 4$ ).

As smoking is strongly associated with DNAm, we conducted a second sensitivity analysis by exploring the associations between alcohol consumption and AgeAccelGrim and AgeAccelPheno in a subset of non-smoking participants (Figure 3 ). The positive associations between alcohol consumption and the two EAA measures remained significant in non-smokers, but the effect size was slightly ( 15\%) attenuated for AgeAccelGrim $(\beta=0.045$ [0.026; 0.061], $p=6.48 \times 10^{-6}$ ) although it remained similar for AgeAccelPheno $\left(\beta=0.074\right.$ [0.043; 0.104], $\left.p=7.74 \times 10^{-6}\right)$.

\section{3 | Testing for the causal influence of alcohol use on accelerated brain age}

Having demonstrated a consistent phenotypic association between alcohol use and accelerated brain age, we used two-sample MR to test a causal relationship between AUD/alcohol consumption (AUDIT-C) and brain age (Figure 4). For AUD, the IVW model was significant $(\beta=0.118$ [0.003; 0.233], $p=0.044 ; \mathrm{mF}=73.744$ [Table S7]) (Figure 4B) but not for AUDIT-C (Figure 4A), suggesting that AUD, but not alcohol consumption levels, has a possible causal effect on accelerated/advanced brain age. However, Steiger filtering suggested that two of the genetic variants explained more variance in the outcome, suggesting some potential for reverse causation (Table S13). Additionally, Radial MR revealed rs570436 ( $Q=4.854$, $p=0.028$ ) as an outlier in the MR analysis of AUD and brain age, and therefore, the analysis was repeated with this SNP excluded. After removing the outlying SNP, the IVW model was no longer significant $(\beta=0.092$ [-0.001; 0.185], $p=0.054)$, suggesting that the

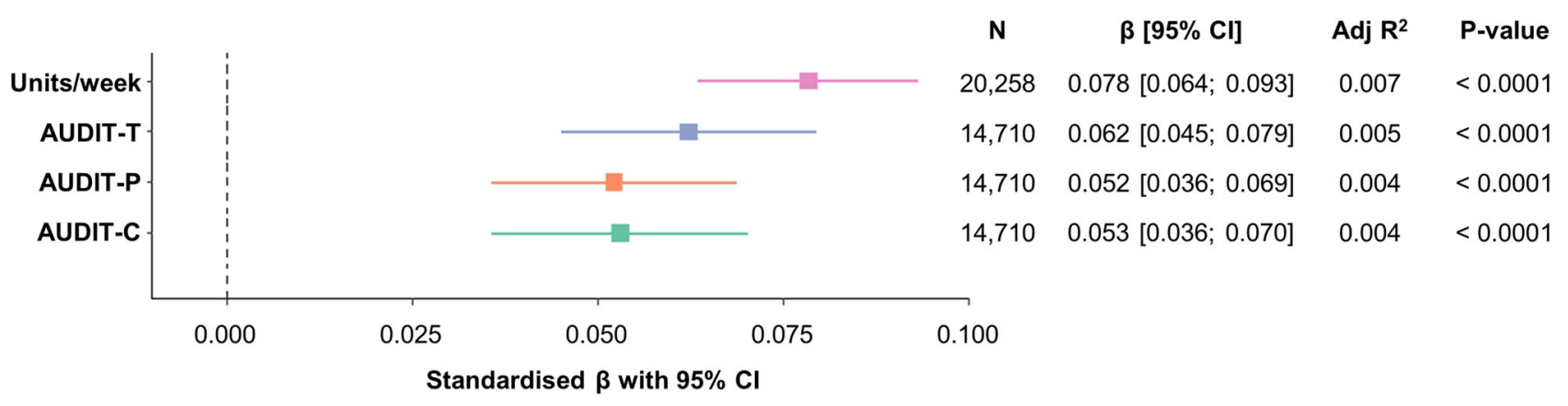

FIGURE 1 Alcohol use is associated with advanced brain age. Linear regression models predicting residual brain age from AUDIT-C, AUDIT-P, AUDIT-T and alcohol units, in current drinkers adjusted for smoking status. Plot shows standardised $\beta$ coefficients with $95 \%$ confidence intervals. $\mathrm{Cl}$, confidence interval 
A EEAA

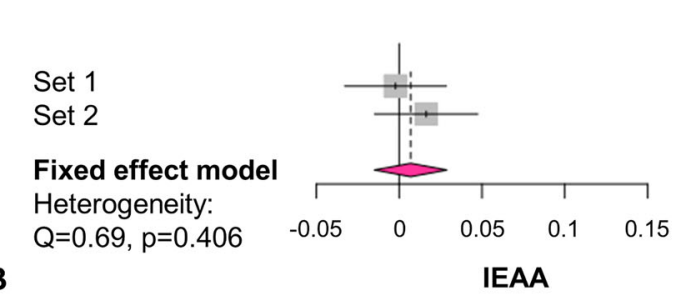

$\begin{array}{ccccc}\boldsymbol{\beta}[\mathbf{9 5 \%} \mathrm{Cl}] & \text { SE } & \text { Weight \% } & \text { p-value } & \begin{array}{c}\text { FDR cor. } \\ \text { p-value }\end{array} \\ -0.002[-0.033 ; 0.029] & 0.016 & 50.7 & 0.887 & 0.989 \\ 0.016[-0.015 ; 0.048] & 0.016 & 49.3 & 0.304 & 0.521 \\ \mathbf{0 . 0 0 7}[-\mathbf{- 0 . 0 1 5 ; 0 . 0 2 9 ]} & & \mathbf{1 0 0 . 0} & \mathbf{0 . 5 3 6} & \mathbf{0 . 6 4 3}\end{array}$

B

IEAA

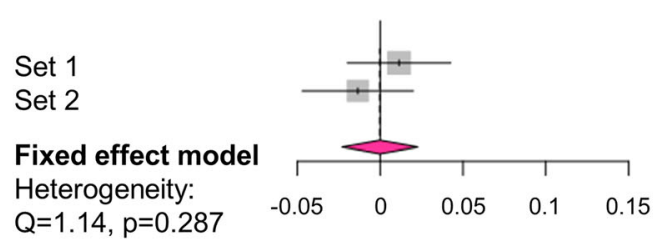

$\begin{array}{ccccc}\boldsymbol{\beta}[\mathbf{9 5 \%} \mathrm{Cl}] & \text { SE } & \text { Weight \% } & \text { p-value } & \begin{array}{c}\text { FDR cor. } \\ \text { p-value }\end{array} \\ 0.011[-0.020 ; 0.043] & 0.016 & 53.6 & 0.474 & 0.632 \\ 0.013[-0.047 ; 0.020] & 0.017 & 46.4 & 0.440 & 0.632 \\ \mathbf{0 . 0 0 7 [ - 0 . 0 2 3 ; 0 . 0 2 3 ]} & & \mathbf{1 0 0 . 0} & \mathbf{0 . 9 8 9} & \mathbf{0 . 9 8 9}\end{array}$

C

AgeAccelGrim

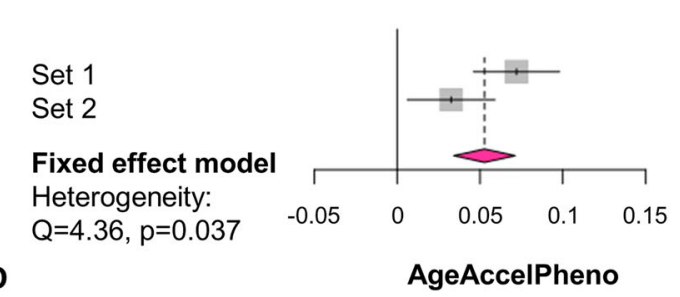

$\begin{array}{ccccc}\boldsymbol{\beta}[95 \% \mathrm{Cl}] & \text { SE } & \text { Weight } \% & \text { p-value } & \begin{array}{c}\text { FDR cor. } \\ \text { p-value }\end{array} \\ 0.072[0.046 ; 0.098] & 0.013 & 51.0 & 5.58 \times 10^{-8} & 2.23 \times 10^{-7} \\ 0.033[0.006 ; 0.059] & 0.014 & 49.0 & 0.016 & 0.032 \\ \mathbf{0 . 0 5 3}[\mathbf{0 . 0 3 4 ; 0 . 0 7 1 ]} & & \mathbf{1 0 0 . 0} & \mathbf{2 . 4 7 \times 1 0 ^ { - 8 }} & \mathbf{1 . 4 8 \times 1 0 ^ { - 7 }}\end{array}$

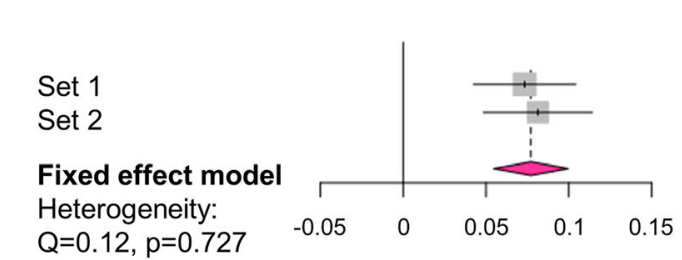

$\begin{array}{ccccc}\boldsymbol{\beta}[\mathbf{9 5 \%} \mathrm{CI}] & \text { SE } & \text { Weight \% } & \text { p-value } & \begin{array}{c}\text { FDR cor. } \\ \text { p-value }\end{array} \\ 0.073[0.043 ; 0.104] & 0.016 & 53.2 & 3.19 \times 10^{-6} & 7.67 \times 10^{-6} \\ 0.081[0.049 ; 0.114] & 0.017 & 46.8 & 1.28 \times 10^{-6} & 3.84 \times 10^{-6} \\ \mathbf{0 . 0 7 7}[\mathbf{0 . 0 5 5} ; \mathbf{0 . 1 0 0}] & & \mathbf{1 0 0 . 0} & \mathbf{1 . 8 2 \times 1 0 ^ { - 1 1 }} & \mathbf{2 . 1 8 \times 1 0 ^ { - 1 0 }}\end{array}$

FIGURE 2 Alcohol consumption is associated with two measures of advanced epigenetic age. Effects of alcohol consumption (units/week) on (A) EEAA, (B) IEAA, (C) AgeAccelGrim and (D) AgeAccelPheno in fully adjusted models. Values on forest plot indicate standardised $\beta$ with $95 \%$ confidence intervals. Models are adjusted for sex, BMI and pack-years in Sets 1 and 2 and relatedness in Set 1 by fitting pedigree information as a random effect in general linear mixed models using advanced restricted maximum likelihood (ASReml) method. Fixed-effects inverse varianceweighted meta-analysis was applied using R package meta to combine the standardised coefficient estimates in Sets 1 and 2 . FDR correction was applied across all models in Sets 1 and 2 and all meta-analysis models (12 models in total). Sample size: $n=4260$ in Set 1, $n=3791$ in Set 2 ( $n=8051$ included in meta-analyses). Cl, confidence interval; EEAA, extrinsic epigenetic age acceleration; FDR, false discovery rate; IEAA, intrinsic epigenetic age acceleration; SE, standard error

outlier was driving the significant result for AUD (Figure S5), although the biological function of this variant is not known, so we cannot be sure whether it acts through alcohol consumption. Together, these results provide limited evidence for a causal effect of genetically instrumented AUD on brain age and no evidence of a causal effect of alcohol use on brain age as measured by AUDIT-C.

\subsection{Testing for the causal influence of alcohol consumption on epigenetic age acceleration}

As we observed, a significant association between alcohol consumption and advanced GrimAge and PhenoAge in the observational analysis, we used two-sample MR methods to test whether these effects might be causal. Although the mean F-statistics suggest that the SNPs included in the analyses are strong genetic instruments $(\mathrm{mF}=79.058$;
Table S7), there was no evidence to suggest a causal effect of alcohol consumption (AUDIT-C) on the two EAA measures (Figure 5). Thus, these results show that in this study, we find no evidence that the association between alcohol consumption and accelerated epigenetic age is causal.

\section{4 | DISCUSSION}

This study represents one of the largest systematic investigations of alcohol use and biological ageing to date and is the first study investigating possible causal relationships between alcohol use and accelerated brain and epigenetic ageing using two-sample MR. We report consistent positive associations between four measures of alcohol use and accelerated brain age as well as alcohol consumption and two measures of DNAm age acceleration (AgeAccelPheno and AgeAccelGrim). MR analyses revealed limited evidence for the causal 
A

AgeAccelGrim

\begin{tabular}{|c|c|c|c|c|c|c|}
\hline & & $\beta[95 \% \mathrm{Cl}]$ & SE & Weight $\%$ & p-value & $\begin{array}{c}\text { FDR cor. } \\
\text { p-value }\end{array}$ \\
\hline Set 1 & 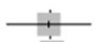 & $0.043[0.017 ; 0.068]$ & 0.013 & 48.5 & $9.16 \times 10^{-4}$ & $1.10 \times 10^{-3}$ \\
\hline Set 2 & 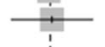 & $0.044[0.020 ; 0.069]$ & 0.012 & 51.5 & $3.58 \times 10^{-4}$ & $7.16 \times 10^{-4}$ \\
\hline $\begin{array}{l}\text { Fixed effect model } \\
\text { Heterogeneity }\end{array}$ & $\sum_{1}^{1}$ & $0.045[0.026 ; 0.061]$ & & 100.0 & $1.08 \times 10^{-6}$ & $6.48 \times 10^{-6}$ \\
\hline
\end{tabular}

B

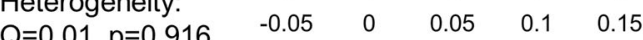

AgeAccelPheno

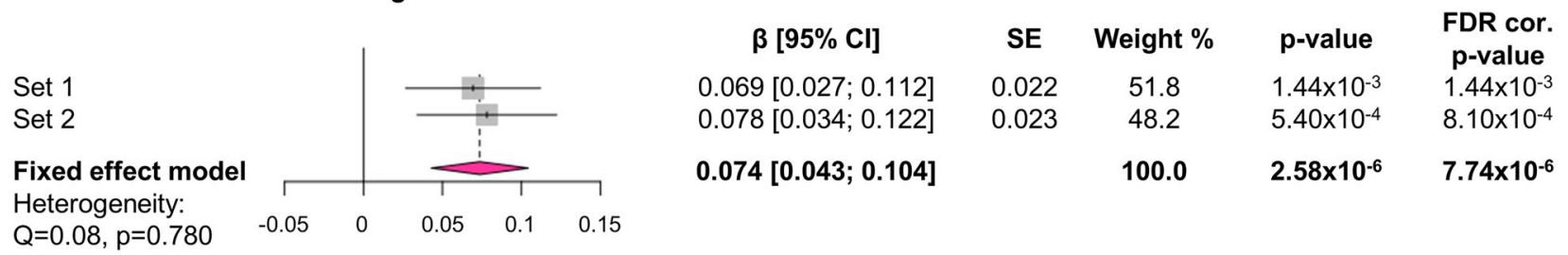

FIGURE 3 Alcohol consumption is associated with advanced GrimAge and PhenoAge in non-smokers. Effects of alcohol consumption (units/week) on (A) AgeAccelGrim and (B) AgeAccelPheno in non-smoking participants. Values on forest plot indicate standardised $\beta$ with $95 \%$ confidence intervals. Models are adjusted for sex and BMI in Sets 1 and 2 and relatedness in Set 1 by fitting pedigree information as a random effect in general linear mixed models using advanced restricted maximum likelihood (ASReml) method. Fixed-effects inverse variance-weighted meta-analysis was applied using R package meta to combine the standardised coefficient estimates in Sets 1 and 2 . FDR correction was applied across all smoking sensitivity models in Sets 1 and 2 and all meta-analysis models (six models in total). Sample size: $n=2207$ in Set $1, n=1998$ in Set 2 ( $n=4205$ included in meta-analyses). Cl, confidence interval; EEAA, extrinsic epigenetic age acceleration; FDR, false discovery rate; IEAA, intrinsic epigenetic age acceleration; SE, standard error

A

\begin{tabular}{|c|c|c|c|c|c|c|}
\hline Exposure & Method & N SNPs & & & $\beta[95 \% \mathrm{Cl}]$ & P-value \\
\hline \multirow[t]{5}{*}{ AUDIT-C } & Inverse variance weighted & 13 & & & $0.045[-0.116 ; 0.207]$ & 0.584 \\
\hline & MR Egger & 13 & & & $0.159[-0.094 ; 0.412]$ & 0.244 \\
\hline & Weighted median & 13 & & & $0.071[-0.099 ; 0.242]$ & 0.414 \\
\hline & Weighted mode & 13 & & & $0.093[-0.101 ; 0.286]$ & 0.366 \\
\hline & & -0.25 & 0.00 & 0.25 & 0.50 & \\
\hline Exposure & Method & N SNPs & & & $\beta[95 \% \mathrm{Cl}]$ & P-value \\
\hline \multirow[t]{5}{*}{ AUD } & Inverse variance weighted & 10 & & & $0.118[0.003 ; 0.233]$ & 0.044 \\
\hline & MR Egger & 10 & & & $0.032[-0.135 ; 0.199]$ & 0.717 \\
\hline & Weighted median & 10 & & & $0.078[-0.031 ; 0.187]$ & 0.162 \\
\hline & Weighted mode & 10 & $\longrightarrow$ & & $0.063[-0.044 ; 0.169]$ & 0.281 \\
\hline & & -0.25 & 0.00 & 0.25 & 0.50 & \\
\hline
\end{tabular}

FIGURE 4 Two-sample Mendelian randomisation analysis provides weak evidence for a causal effect of AUD on brain age acceleration. (A) Two sample Mendelian randomisation of AUDIT-C on brain age. (B) Two-sample Mendelian randomisation of AUD on brain age. Data on the genetic association with AUDIT-C and AUD were extracted from Kranzler et al. ${ }^{22}$ Summary statistics for these SNPs were extracted from a novel GWAS of brain age (see Section 2). AUD, alcohol use disorder; Cl, confidence interval; N SNP, number of SNPs included in the MR analysis

effect of AUD on accelerated brain age, although there was no evidence to suggest a causal link between levels of alcohol consumption and brain or epigenetic ageing.

In the present study, we demonstrate a positive association between four measures of alcohol use and a metric of accelerated brain ageing, derived from structural MRI. We show associations with problematic alcohol use (AUDIT-P), alcohol consumption (AUDIT-C) and total AUDIT scores, with the strongest phenotypic association found for alcohol consumption in units/week. These results are consistent with previous investigations showing that brain changes associated with ageing are more pronounced in individuals with higher levels of alcohol use $\mathrm{e}^{2}$ and with previous reports of advanced 
A

\begin{tabular}{|c|c|c|c|c|c|c|c|c|c|c|}
\hline Outcome & Method & N SNPs & & & $\beta[95 \% \mathrm{Cl}]$ & & & & $\beta[95 \% \mathrm{Cl}]$ & $P$-value \\
\hline \multirow[t]{5}{*}{ AgeAccelGrim } & Inverse variance weighted & 13 & & 1 & & & & & $-0.195[-0.577 ; 0.188]$ & 0.318 \\
\hline & MR Egger & 13 & & & & & & & $0.058[-0.612 ; 0.728]$ & 0.868 \\
\hline & Weighted median & 13 & & - & & & & & $-0.111[-0.656 ; 0.435]$ & 0.691 \\
\hline & Weighted mode & 13 & & 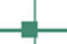 & & & & & $-0.022[-0.643 ; 0.600]$ & 0.947 \\
\hline & & -1.0 & -0.5 & 0.0 & 0.5 & 1.0 & 1.5 & 2.0 & & \\
\hline Outcome & Method & N SNPs & & & $\beta[95 \% \mathrm{Cl}]$ & & & & $\beta[95 \% \mathrm{Cl}]$ & P-value \\
\hline \multirow[t]{5}{*}{ AgeAccelPheno } & Inverse variance weighted & 13 & & - & - & & & & $0.177[-0.559 ; 0.912]$ & 0.638 \\
\hline & MR Egger & 13 & & & 늘 & & — & 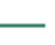 & $0.760[-0.563 ; 2.083]$ & 0.284 \\
\hline & Weighted median & 13 & & & 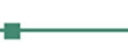 & & & & $0.303[-0.435 ; 1.041]$ & 0.421 \\
\hline & Weighted mode & 13 & & & - & $\ldots$ & & & $0.365[-0.476 ; 1.196]$ & 0.407 \\
\hline & & -1.0 & -0.5 & 0.0 & 0.5 & 1.0 & 1.5 & 2.0 & & \\
\hline
\end{tabular}

FIGURE 5 Two-sample Mendelian randomisation analysis shows no evidence for causal effect of alcohol consumption on measures of epigenetic age acceleration: (A) AgeAccelGrim and (B) AgeAccelPheno. Data on the genetic association with alcohol use (AUDIT-C) were extracted from Kranzler et al. ${ }^{22}$ Summary statistics for these SNPs were extracted from GWASs for AgeAccelGrim and AgeAccelPheno conducted by McCartney et al. ${ }^{21}$ rs185177474 was not available in AgeAccelGrim and AgeAccelPheno summary statistics; thus, rs 151242810 was used as a $\operatorname{proxy}\left(R^{2}=0.976\right.$; see Section 2). Cl, confidence interval; N SNP, number of SNPs included in the MR analysis

brain age relative to peers in individuals who consume alcohol more frequently. ${ }^{15,16}$ The present study provides evidence that brain changes in response to excessive alcohol use resemble an early ageing process.

We further demonstrate a positive association between alcohol consumption and accelerated DNAm PhenoAge and GrimAge, thus expanding on the results of Fiorito et al. ${ }^{18}$ who showed positive association between alcohol consumption levels and accelerated PhenoAge. However, we do not replicate previous findings showing associations between self-reported alcohol consumption levels and accelerated DNAm age derived from Hannum and Horvath clocks, ${ }^{12,18}$ which could be attributed to differences in study populations, ${ }^{12}$ differences in the use of alcohol use variables in the models ${ }^{18}$ and the use of different DNAm arrays. ${ }^{12,18}$ Furthermore, the first-generation clocks (Horvath and Hannum) were designed for the Illumina $27 \mathrm{k}$ and $450 \mathrm{k}$ arrays, whereas PhenoAge and GrimAge were developed using overlapping CpG sites on the Illumina $450 \mathrm{~K}$ and EPIC arrays, and there are reports suggesting inaccurate Hannum and Horvath age estimations when using the EPIC array. ${ }^{45}$ Importantly, the novel PhenoAge and GrimAge estimators are shown to be stronger predictors of mortality and lifestyle factors, including alcohol use, ${ }^{18,31,32}$ which could be explained by the inclusion of CpG sites associated with biomarkers of physiological dysregulation, disease and mortality, whereas Hannum and Horvath clocks were designed to predict chronological age.

A major strength of the current study is the use of data from both UKB and GS:SFHS, which enabled association studies to be conducted in much larger samples from single cohorts compared with previous reports ( $n=20,258$ for brain ageing in the current study compared with $n=14,701,{ }^{16} n=12,115^{15}$ and $n=527^{2}$ in previous studies; $n=8051$ for the DNAm ageing in the current study compared with $n=836,{ }^{19} n=3687^{12}$ and $n=16,245^{18}$ [from 18 different cohorts with each between 174 and 2817 participants] in previous studies). An additional strength is the range of measurements enabling a systematic assessment of the association of four different self-reported alcohol use measures with brain age acceleration as well as the association between alcohol consumption and four different measures of EAA.

Using two-sample MR, we report limited evidence suggesting a causal link between the diagnosis of AUD and accelerated brain ageing, but no evidence for higher alcohol consumption causing accelerated brain or epigenetic ageing. We replicate and expand on recent findings showing significant genetic correlations between alcoholrelated phenotypes and epigenetic ageing, but no causal relationship between alcohol use frequency and EAA measures as assessed by MR. ${ }^{21}$ This suggests that the phenotypic association may arise from confounding factors (e.g. other harmful lifestyle factors) that have directional effects on both alcohol use and biological ageing. High levels of psychiatric comorbidity with $\mathrm{AUD}^{46}$ represent another possible confounder. For example, schizophrenia, ${ }^{47}$ major depressive disorder ${ }^{48,49}$ and post-traumatic stress disorder ${ }^{50}$ are associated with accelerated brain and/or epigenetic ageing. Furthermore, sensitivity analyses revealed that some of the genetic variants used here for alcohol use explain more variance in the outcome (brain or DNAm ageing), suggesting potential reverse causation. Finally, the Million Veteran Programme sample used for the GWAS of AUD and alcohol consumption comprises predominantly male armed forces veterans. ${ }^{22}$ Thus, it may not be representative of the whole population, as there is evidence that alcohol use and AUD have higher prevalence in males ${ }^{1}$ and genetic mechanisms might differ between sexes. Additionally, 
there are differences in the phenotypes used in the current study and the ones used in the GWAS for AUD and alcohol consumption by Kranzler et al. $^{22}$ (e.g. clinically diagnosed AUD vs AUDIT-P). Future longitudinal studies combined with more experimental approaches could help elucidate the mechanisms linking alcohol use with biological ageing and their interactions.

Several other limitations need to be addressed when interpreting these findings. First, this study relied on self-reported measures of alcohol use that might be inaccurate due to response biases. Whereas there are traditionally few alternatives, the validation of approaches such as estimation of alcohol drinking via the generation of a composite score for alcohol use from DNAm data ${ }^{19,51}$ or other biological data (e.g. liver enzyme levels as investigated in association with EAA previously ${ }^{17}$ ) could help to overcome the need to rely on self-reported measures. Second, although we investigated the associations between problematic alcohol use (AUDIT-P) and brain ageing, we were unable to conduct a similar evaluation in association with DNAm ageing measures. Additionally, different drinking patterns may be important. It was recently suggested that the relationship between educational attainment and adverse health outcomes is mediated by specific patterns of alcohol use, such as binge drinking, rather than total alcohol consumption. ${ }^{52}$ Thus, the focus on overall alcohol consumption in the present study might preclude the detection of a causal relationship. Finally, we investigated the associations between alcohol use and brain ageing or blood DNAm ageing in two separate large cohorts. It would be of interest in future studies to investigate the associations between alcohol use and the two types of biological ageing measures in the same individuals and evaluate the relationship between brain and DNAm ageing.

To conclude, in one of the largest studies on the relationship between alcohol use and biological ageing to date, and a first investigation of the causal relationship between the two using MR, we report a consistent association between higher levels of alcohol consumption and accelerated biological ageing. The present study found very limited evidence that the diagnosis of AUD might be causally linked to accelerated brain ageing; however, these results need to be interpreted with caution as they are driven by an outlier variant with unknown biological function and there is some limited evidence of potential reverse causation. Using two-sample MR, we additionally found no evidence for a causal link between alcohol consumption levels and biological ageing indicated by brain or DNAm ageing measures. The positive phenotypic associations between alcohol consumption and brain and epigenetic ageing add to the body of literature suggesting that alcohol use is associated with biomarkers predicting early ageing and mortality; however, the precise nature of this relationship remains to be identified in future studies.

\section{ACKNOWLEDGEMENTS}

This research was funded in whole, or in part, by the Wellcome Trust (108890/Z/15/Z). For the purpose of open access, the author has applied a CC BY public copyright licence to any Author Accepted Manuscript version arising from this submission. This work was supported by a Wellcome Trust Strategic Award 'Stratifying
Resilience and Depression Longitudinally' (STRADL) (104036/Z/14/ Z). Generation Scotland received core support from the Chief Scientist Office of the Scottish Government Health Directorates (CZD/16/6) and the Scottish Funding Council (HR03006). Genotyping of the GS:SFHS samples was funded by the Medical Research Council UK and the Wellcome Trust (Wellcome Trust Strategic Award [STRADL]; reference as above). DNAm profiling of the GS:SFHS samples was funded by the Wellcome Trust Strategic Award (104036/Z/14/Z) with additional funding from a 2018 NARSAD Young Investigator Grant from the Brain and Behavior Research Foundation (27404). The UK Biobank core activities and imaging study were funded primarily by the Wellcome Trust and the MRC, as well as the Department of Health and the National Institute for Health Research (NIHR), the Scottish and Welsh Governments, the British Heart Foundation and Cancer Research UK. SMKB and $\mathrm{KV}$ are funded by the Wellcome Trust Translational Neuroscience PhD Programme at the University of Edinburgh (108890/Z/15/Z). $J H C$ is supported by a UK Research and Innovation Fellowship (MR/R024790/2). SRC received support from the UK MRC (MR/R024065/1) and US National Institutes of Health (R01AG054628). DLM and REM were supported by Alzheimer's Research UK Major project grant (ARUK-PG2017B-10). REW works in a unit that receives funding from the University of Bristol and the UK Medical Research Council (MC_UU_00011/1 and MC_UU_00011/3). Figures were made using BioRender and R packages ggplot2 and ggpubr. FUMA analysis was carried out by Miruna Carmen Barbu.

\section{CONFLICT OF INTEREST}

AMM reports grants from The Sackler Trust, grants from Eli Lilly and grants from Janssen outside the submitted work. JHC is a shareholder in and scientific advisor to Brain Key, a medical image analysis software company. The remaining authors declare no conflicts of interest. This manuscript has been uploaded as a preprint to MedRxiv.

\section{AUTHOR CONTRIBUTIONS}

SMKB and KV performed data analysis, wrote the manuscript and contributed to the study concept and design. TCK was responsible for the study design and concept and assisted with interpretation of findings. REW assisted with data analysis. REW and HCW contributed to the study concept and design and assisted with interpretation of findings. TCK, REW and HCW supervised data analysis and writing of the manuscript. LDN, MAH, REM, DLM, RMW and KLE performed data preprocessing. LDN, MAH, JHC, SRC, REM, DLC, RMW, AMM, KLE, $\mathrm{HCW}$ and TKC provided critical revision of the manuscript for important intellectual content. All authors critically reviewed content and approved the final version for publication.

\section{DATA AVAILABILITY STATEMENT}

According to the terms of consent for GS:SFHS, access to data must be reviewed by the GS Access Committee (access@ generationscotland.org). Details concerning access to UK Biobank data can be found in the link provided: https://www.ukbiobank.ac.uk/ 
principles-of-access/. Summary statistics from GWAS of AgeAccelPheno and AgeAccelGrim are available here: https://doi.org/ $10.7488 / \mathrm{ds} / 2834$. Summary statistics from GWAS of brain age acceleration are available here: https://doi.org/10.7488/ds/2956.

\section{ORCID}

Sunniva M. K. Bøstrand (D) https://orcid.org/0000-0003-1689-8583 Kadi Vaher (D) https://orcid.org/0000-0002-0733-2505

\section{REFERENCES}

1. Griswold MG, Fullman N, Hawley C, et al. Alcohol use and burden for 195 countries and territories, 1990-2016: a systematic analysis for the global burden of disease study 2016. Lancet. 2018;392(10152): 1015-1035. https://doi.org/10.1016/S0140-6736(18)31310-2

2. Topiwala A, Allan CL, Valkanova V, et al. Moderate alcohol consumption as risk factor for adverse brain outcomes and cognitive decline: longitudinal cohort study. BMJ. 2017;257:j2353. https://doi.org/10. 1136/bmj.j2353

3. Laramée $P$, Leonard $S$, Buchanan-Hughes $A$, Warnakula $S$, Daeppen J-B, Rehm J. Risk of all-cause mortality in alcohol-dependent individuals: a systematic literature review and meta-analysis. EBIOM. 2015;2(10):1394-1404. https://doi.org/10.1016/j.ebiom.2015. 08.040

4. Rehm J, Gmel GE, Gmel G, et al. The relationship between different dimensions of alcohol use and the burden of disease-an update. Addiction. 2017;112(6):968-1001. https://doi.org/10.1111/add. 13757

5. López-Otín C, Blasco MA, Partridge L, Serrano M, Kroemer G. The hallmarks of aging. Cell. 2013;153(6):1194-1217. https://doi.org/10. 1016/j.cell.2013.05.039

6. Cole JH, Poudel RPK, Tsagkrasoulis D, et al. Predicting brain age with deep learning from raw imaging data results in a reliable and heritable biomarker. Neuroimage. 2017;163:115-124. https://doi.org/10.1016/ j.neuroimage.2017.07.059

7. Sprott RL. Biomarkers of aging and disease: introduction and definitions. Exp Gerontol. 2010;45(1):2-4. https://doi.org/10.1016/j.exger. 2009.07.008

8. Cole $\mathrm{JH}$, Franke K. Predicting age using neuroimaging: innovative brain ageing biomarkers. Trends Neurosci. 2017;40(12):681-690. https://doi.org/10.1016/j.tins.2017.10.001

9. Cole JH, Ritchie SJ, Bastin ME, et al. Brain age predicts mortality. Mol Psychiatry. 2018;23(5):1385-1392. https://doi.org/10.1038/mp. 2017.62

10. Horvath S. DNA methylation age of human tissues and cell types. Genome Biol. 2013;14(10):3156. https://doi.org/10.1186/gb-201314-10-r115

11. Marioni RE, Shah S, McRae AF, et al. DNA methylation age of blood predicts all-cause mortality in later life. Genome Biol. 2015;16(1):25. https://doi.org/10.1186/s13059-015-0584-6

12. Quach A, Levine ME, Tanaka T, et al. Epigenetic clock analysis of diet, exercise, education, and lifestyle factors. Aging (Albany NY). 2017; 9(2):419-437. https://doi.org/10.18632/aging.101168

13. Marioni RE, Shah S, Mcrae AF, et al. The epigenetic clock is correlated with physical and cognitive fitness in the Lothian birth cohort 1936. Int J Epidemiol. 2015;44(4):1388-1396. https://doi.org/10.1093/ije/ dyu277

14. Hillary RF, Stevenson AJ, Cox SR, et al. An epigenetic predictor of death captures multi-modal measures of brain health. Mol Psychiatry. 2019;1-11. https://doi.org/10.1038/s41380-019-0616-9

15. Ning K, Zhao L, Matloff W, Sun F, Toga AW. Association of relative brain age with tobacco smoking, alcohol consumption, and genetic variants. Sci Rep. 2020;10(1):1-10. https://doi.org/10.1038/s41598019-56089-4
16. Cole JH. Multimodality neuroimaging brain-age in UK biobank: relationship to biomedical, lifestyle, and cognitive factors. Neurobiol Aging. 2020;92:34-42. https://doi.org/10.1016/j.neurobiolaging. 2020.03.014

17. Luo A, Jung J, Longley $M$, et al. Epigenetic aging is accelerated in alcohol use disorder and regulated by genetic variation in APOL2. Neuropsychopharmacology. 2020;45(2):327-336. https://doi.org/10. 1038/s41386-019-0500-y

18. Fiorito G, McCrory C, Robinson O, et al. Socioeconomic position, lifestyle habits and biomarkers of epigenetic aging: a multi-cohort analysis. Aging (Albany NY). 2019;11(7):2045-2070. https://doi.org/ 10.18632/aging.101900

19. Beach SRH, Dogan MV, Lei M-K, et al. Methylomic aging as a window onto the influence of lifestyle: tobacco and alcohol use alter the rate of biological aging. J am Geriatr Soc. 2015;63(12):2519-2525. https:// doi.org/10.1111/jgs.13830

20. Zhang $\mathrm{H}$, Gelernter J. Review: DNA methylation and alcohol use disorders: progress and challenges. Am J Addict. 2017;26(5):502-515. https://doi.org/10.1111/ajad.12465

21. McCartney DL, Min JL, Richmond RC, et al. Genome-wide association studies identify 137 genetic loci for DNA methylation biomarkers of aging. Genome Biol. 2021;22(1):194. https://doi.org/10.1186/ S13059-021-02398-9

22. Kranzler HR, Zhou H, Kember RL, et al. Genome-wide association study of alcohol consumption and use disorder in 274,424 individuals from multiple populations. Nat Commun. 2019;10(1):1499. https:// doi.org/10.1038/s41467-019-09480-8

23. Clarke T-K, Adams MJ, Davies G, et al. Genome-wide association study of alcohol consumption and genetic overlap with other health-related traits in UK biobank ( $N=112$ 117). Mol Psychiatry. 2017;22(10):1376-1384. https://doi.org/10.1038/mp. 2017.153

24. Smith GD, Hemani G. Mendelian randomization: genetic anchors for causal inference in epidemiological studies. Hum Mol Genet. 2014; 23(R1):R89-R98. https://doi.org/10.1093/hmg/ddu328

25. Miller KL, Alfaro-Almagro F, Bangerter NK, et al. Multimodal population brain imaging in the UK biobank prospective epidemiological study. Nat Neurosci. 2016;19(11):1523-1536. https://doi.org/10. 1038/nn.4393

26. Saunders JB, Aasland OG, Babor TF, de la Fuente JR, Grant M. Development of the alcohol use disorders identification test (AUDIT): WHO collaborative project on early detection of persons with harmful alcohol consumption-II. Addiction. 1993;88(6):791-804. https:// doi.org/10.1111/j.1360-0443.1993.tb02093.x

27. Smith $B H$, Campbell $A$, Linksted $P$, et al. Cohort profile: generation Scotland: Scottish family health study (GS:SFHS). The study, its participants and their potential for genetic research on health and illness. Int J Epidemiol. 2013;42(3):689-700. https://doi.org/10.1093/ ije/dys084

28. Barbu MC, Shen X, Walker RM, et al. Epigenetic prediction of major depressive disorder. Mol Psychiatry. 2020. https://doi.org/10.1038/ s41380-020-0808-3

29. Pidsley R, Y Wong CC, Volta M, Lunnon K, Mill J, Schalkwyk LC. A data-driven approach to preprocessing Illumina $450 \mathrm{~K}$ methylation array data. BMC Genomics. 2013;14(1):293. https://doi.org/10.1186/ 1471-2164-14-293

30. Hannum G, Guinney J, Zhao L, et al. Genome-wide methylation profiles reveal quantitative views of human aging rates. Mol Cell. 2013; 49(2):359-367. https://doi.org/10.1016/j.molcel.2012.10.016

31. Lu AT, Quach A, Wilson JG, et al. DNA methylation GrimAge strongly predicts lifespan and healthspan. Aging (Albany NY). 2019;11(2): 303-327.

32. Levine ME, Lu AT, Quach A, et al. An epigenetic biomarker of aging for lifespan and healthspan. Aging (Albany NY). 2018;10(4):573-591. https://doi.org/10.18632/aging.101414 
33. Horvath S, Gurven M, Levine ME, et al. An epigenetic clock analysis of race/ethnicity, sex, and coronary heart disease. Genome Biol. 2016; 17(1):171. https://doi.org/10.1186/s13059-016-1030-0

34. Klemera P, Doubal S. A new approach to the concept and computation of biological age. Mech Ageing Dev. 2006;127(3):240-248. https://doi.org/10.1016/J.MAD.2005.10.004

35. R Core Team. R: a language and environment for statistical computing. R Foundation for Statistical Computing. 2020. https://www.rproject.org/

36. Balduzzi S, Rücker G, Schwarzer G. How to perform a meta-analysis with R: a practical tutorial. Evid Based Ment Health. 2019;22(4): 153-160. https://doi.org/10.1136/ebmental-2019-300117

37. Hemani G, Zheng J, Elsworth B, et al. The MR-base platform supports systematic causal inference across the human phenome. Elife. 2018;7: e34408. https://doi.org/10.7554/eLife.34408

38. Watanabe K, Taskesen E, Van Bochoven A, Posthuma D. Functional mapping and annotation of genetic associations with FUMA. Nat Commun. 2017;8(1):1-11. https://doi.org/10.1038/s41467-01701261-5

39. Zheng J, Erzurumluoglu AM, Elsworth BL, et al. LD hub: a centralized database and web interface to perform LD score regression that maximizes the potential of summary level GWAS data for SNP heritability and genetic correlation analysis. Bioinformatics. 2017;33(2):272-279. https://doi.org/10.1093/bioinformatics/btw613

40. Bowden J, Fabiola Del Greco M, Minelli C, Smith GD, Sheehan NA Thompson JR. Assessing the suitability of summary data for two-sample Mendelian randomization analyses using MR-Egger regression: the role of the I 2 statistic. Int J Epidemiol. 2016;45(6): 1961-1974. https://doi.org/10.1093/ije/dyw220

41. Pasman JA, Verweij $\mathrm{KJH}$, Gerring Z, et al. GWAS of lifetime cannabis use reveals new risk loci, genetic overlap with psychiatric traits, and a causal effect of schizophrenia liability. Nat Neurosci. 2018;21(9): 1161-1170. https://doi.org/10.1038/s41593-018-0206-1

42. Hemani G, Bowden J, Haycock P, et al. Automating Mendelian randomization through machine learning to construct a putative causal map of the human phenome. bioRxiv. 2017. https://doi.org/10.1101/ 173682

43. Verbanck M, Chen C-Y, Neale B, Do R. Detection of widespread horizontal pleiotropy in causal relationships inferred from Mendelian randomization between complex traits and diseases. Nat Genet. 2018; 50(5):693-698. https://doi.org/10.1038/s41588-018-0099-7

44. Bowden J, Spiller W, Fabiola Del Greco M, et al. Improving the visualization, interpretation and analysis of two-sample summary data Mendelian randomization via the Radial plot and Radial regression. Int J Epidemiol. 2017;47(4):1264-1278. https://doi.org/10.1093/ije/ dyy101

45. Dhingra R, Kwee LC, Diaz-Sanchez D, et al. Evaluating DNA methylation age on the Illumina MethylationEPIC Bead Chip. PLoS ONE. 2019;14(4):e0207834. https://doi.org/10.1371/journal.pone. 0207834
46. Castillo-Carniglia A, Keyes KM, Hasin DS, Cerdá M. Psychiatric comorbidities in alcohol use disorder. Lancet Psychiatry. 2019;6(12): 1068-1080. https://doi.org/10.1016/S2215-0366(19)30222-6

47. Nenadić I, Dietzek M, Langbein K, Sauer H, Gaser C. BrainAGE score indicates accelerated brain aging in schizophrenia, but not bipolar disorder. Psychiatry Res - Neuroimaging. 2017;266:86-89. https://doi. org/10.1016/j.pscychresns.2017.05.006

48. Han LKM, Dinga R, Hahn T, et al. Brain aging in major depressive disorder: results from the ENIGMA major depressive disorder working group. Mol Psychiatry. 2020;1-16. https://doi.org/10.1038/s41380020-0754-0

49. Han LKM, Aghajani M, Clark SL, et al. Epigenetic aging in major depressive disorder. Am J Psychiatry. 2018;175(8):774-782. https:// doi.org/10.1176/appi.ajp.2018.17060595

50. Boks MP, van Mierlo HC, Rutten BP, et al. Longitudinal changes of telomere length and epigenetic age related to traumatic stress and post-traumatic stress disorder. Psychoneuroendocrinology. 2015;51: 506-512. https://doi.org/10.1016/j.psyneuen.2014.07.011

51. Philibert RA, Penaluna B, White $T$, et al. A pilot examination of the genome-wide DNA methylation signatures of subjects entering and exiting short-term alcohol dependence treatment programs. Epigenetics. 2014;9(9):1212-1219. https://doi.org/10.4161/epi.32252

52. Rosoff DB, Clarke TK, Adams MJ, et al. Educational attainment impacts drinking behaviors and risk for alcohol dependence: results from a two-sample Mendelian randomization study with $\sim 780,000$ participants. Mol Psychiatry. 2019;26(4):1119-1132. https://doi.org/ 10.1038/s41380-019-0535-9

\section{DATA REFERENCES}

Bøstrand SMK, Vaher K, de Nooij L, et al. Associations between alcohol use and accelerated biological ageing. Edinburgh DataShare. 2020 https://doi.org/10.7488/ds/2956

McCartney D, Min J, Richmond R, et al. Genome-wide association studies identify 137 loci for DNA methylation biomarkers of aging. Edinburgh DataShare. 2020. https://doi.org/10.7488/ds/2834

\section{SUPPORTING INFORMATION}

Additional supporting information may be found in the online version of the article at the publisher's website.

How to cite this article: Bøstrand SMK, Vaher K, de Nooij L, et al. Associations between alcohol use and accelerated biological ageing. Addiction Biology. 2021;e13100. doi: 10.1111/adb.13100 